\title{
Theory of Diffraction in Microwave Interferometry
}

\author{
D. M. Kerns and E. S. Dayhoff ${ }^{1}$
}

(August 25, 1959)

\begin{abstract}
Microwave Michelson and Fabry-Perot interferometers are respectively considered as instances of: (1) A "reflection system", consisting of a radiating-receiving system and a reflecting object (e.g., a finite mirror); and (2) a "transmission system", consisting of a radiating system and a receiving system with an object (e.g., a Fabry-Perot etalon) interposed. The basic theoretical objective is the calculation of the amplitude and phase of the (time-harmonic) received signal in the systems considered. The electromagnetic field in space transmission paths is represented in terms of continuous angular spectra of vectorial plane waves, and the elements of the systems are described by means of suitable tensor scattering matrices (having both discrete and continuous indices). Needed scattering matrices are considered known; relationships to experimentally determinable data are outlined. The general case of either the reflection or transmission system is soluble formally in terms of a series of integrals stemming from the Liouville-Neumann series solution of certain integral equations. Formulas are obtained for models of the Michelson and FabryPerot instruments with arbitrary radiating and receiving characteristics. The theory and various features of the instruments considered, including Fresnel-region (or quasi-optical) behavior, are illustrated by means of examples obtained by choosing relatively simple and rather hypothetical analytical expressions for the radiating and receiving characteristics.
\end{abstract}

\section{Introduction}

Microwave versions of the Michelson and the Fabry-Perot interferometers are being studied and developed at the National Bureau of Standards with a view to high-accuracy measurements of wavelengths, lengths, and the speed of light [1]. ${ }^{2}$ The data provided by these instruments are, however, subject to corrections for effects which are completely negligible in optical interferometry but significant in the microwave region. In this work - in contrast to the corresponding optical case the principal such effect results from the wavelength not being negligibly small relative to the dimensions of the apparatus, and consequently the effects of diffraction upon the wavelength as observed by the instrument must be carefully considered in order to exploit fully the potential accuracy of the method. In this paper we consider these effects and present analytical tools suitable for making a correction to the apparert wavelength [2]. This should make possible the use of Fresnel region microwave interferometry for work of the highest accuracy.

For the purposes of our analysis the pertinent aspects of the class of microwave Michelson interferometers are represented by a "general reflection system." Similarly, the microwave Fabry-Perot instruments are represented by a "general transmission system." (These basic arrangements are described more fully below.) Inasmuch as we wish to provide a theory inherently capable of dealing with high-accuracy experiments, a considerable

\footnotetext{
1 Present address: U.S. Naval Ordnance Laboratory, Silver Spring, Md.
2 Figures in brackets indicate the literature references at the end of this paper.
}

degree of generality is required to avoid over-idealization. It is interesting that the required generality is such that the general reflection system could equally well represent a system consisting of radar and target, for example; similarly, the general transmission system could represent a point-to-point communication system. In view of the generality of the basic arrangements it seems likely that applications of the theory will also be found in problems other than the ones that motivated this work.

The specific theoretical objective is the calculation of the amplitude and phase of the received signal in the systems considered. Theoretical expressions for the received signal will contain, at least implicitly, all available information regarding diffraction errors, intensities, etc. The analytical technique employed involves a transducer point-of-view, scatteringmatrix formalism and constitutes a generalization of a part of the theory of waveguides and waveguide junctions.

The arrangements to be considered involve radiating, receiving, and radiating-receiving systems as elements, which we shall occasionally refer to as "terminals" or as "terminal apparatus." Such an apparatus is shown schematically in figure 1 . The left-hand block in the figure may contain sources of power, detectors, and any other required auxiliary equipment, all of which is assumed to be shielded. This equipment is connected by means of waveguide to a waveguide-space transducer, represented by the triangular symbol in the figure. For simplicity in the formulation it will be assumed that the waveguide is of a self-enclosed or shielded type. The arrangement and details of the apparatus are highly arbitrary, an essential but not very restrictive 


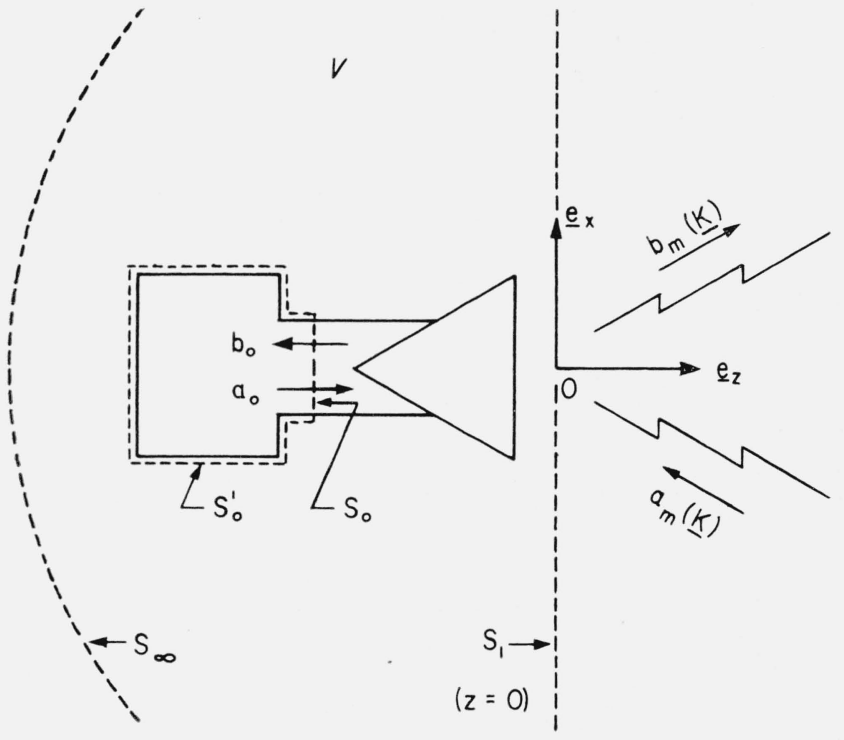

FIGURE 1. Schematic radiating-receiving system.

condition being that the transducer proper possesses the property of linearity with respect to electromagnetic fields. (Details of the statement of hypotheses are given in sec. $2, \mathrm{~A}$.)

In one of the basic arrangements considered - the general reflection system, illustrated in figure 2radiation from a radiating-receiving system is reflected or scattered by a general reflecting object at a variable distance $d$ and is partly received (and partly reflected or scattered) by the same system. The Michelson interferometer can be considered in general terms as a reflection system in which the received signal is observed, as a function of $d$, by causing it to interfere with a suitable reference signal and detecting the resultant amplitude.

In the laboratory version of the Michelson interferometer being considered in connection with the present work, the radiation forms a well-defined beam (within its Fresnel zone), the reflecting object

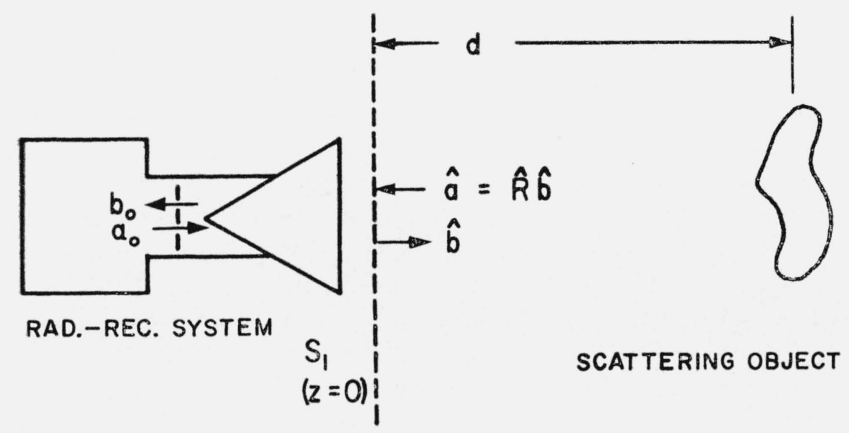

Figure 2. Reflection system.

If the scattering object is a finite mirror, for example, the arrangement represents a microwave Michelson interferometer. is a large but finite mirror placed well within the Fresnel zone of the beam, and multiple reflections between the mirror and the radiating-receiving system are relatively unimportant. It may be said that the instrument is operated in the Fresnel or quasi-optical region. This mode of operation implies the inequalities $a \gg \lambda$ and $\mathrm{d} \ll a^{2} / \lambda$, where $a$ is a measure of the cross section of the beam and $\lambda$ is the free-space wavelength corresponding to the frequency of operation. Under these conditions the radiation in the space between radiator and mirror approximates a homogeneous, plane standing-wave and the received signal is approximately proportional to $\exp (2 i k d)$, where $k=2 \pi / \lambda$.

The second basic arrangement considered is illustrated in figure 3 . Here a structure or object that in general may reflect, transmit, and scatter is interposed between a radiating and a receiving system. If the interposed object is some form of Fabry-Perot "etalon" [3], the arrangement represents a general form of the Fabry-Perot interferometer. In this type of instrument the received signal exhibits amplitude variations sharply dependent on the spacing of the plates of the etalon, and this is attributable to interference among components of the radiation having experienced $0,1,2, \ldots$ reflections between the plates.
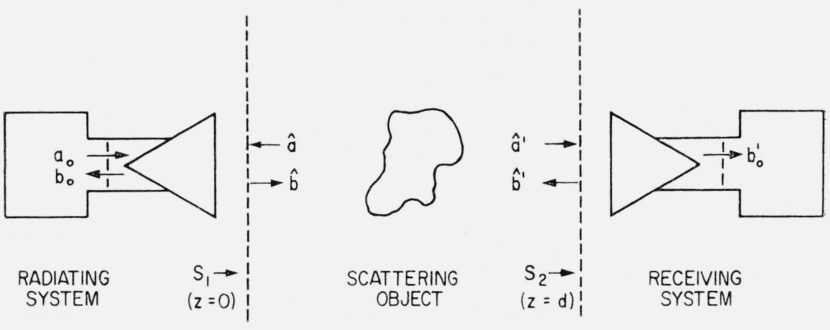

Figure 3. Transmission system.

If the scattering object is a suitable form of Fabry-Perot etalon, the arrangement represents a microwave version of the Fabry-Perot interferometer.

In the laboratory version of the Fabry-Perot being considered in connection with the present work, the instrument is a Fresnel-region instrument in the sense explained above, and multiple reflections, except between the plates of the etalon, are again relatively unimportant.

In the theoretical approach used in the present paper, the (vectorial) electromagnetic field in a space transmission path is represented as the superposition of an angular distribution or spectrum of (vectorial) plane waves. Such a spectrum is in general determined not only by the free-space radiation characteristics of a radiator, but also by the effects of all scattering elements involved (the radiator itself would be such an element, for example, in the case of a Michelson instrument with appreciable multiple reflections). Although the use of the plane-wave representation of the fields is suggested by the close approximation to a single plane wave that might exist in a Fresnel-region instrument, it is hardly 
necessary to remark that in a practical case even the unmodified free-space radiation spectrum is likely to be extremely complicated in detail. This is because of such things as edge effects, geometrical imperfections, and limitations in the design and construction of lenses and horns.

The concept of the plane-wave resolution of the field permits one to form a useful qualitative picture of the origin of an effective wavelength. Consider, for example, a Michelson instrument. Each elementary wave in the spectrum whose normal makes an angle $\theta$ with the line along which translation of the mirror is measured has an effective wavelength $\lambda \sec \theta$; the resultant effective wavelength can be thought of as a kind of average of the elementary contributions over the existing spectrum. Thus one might anticipate, for example, that the resultant effective wavelength should rather generally tend to be greater than the free-space wavelength.

A main section of this paper, section 2, is devoted to establishing a scattering matrix formalism for the description of radiating-receiving systems of the general type considered. In this connection it is helpful - at least to one acquainted with the theory of waveguide junctions - to regard the space side of the waveguide-space transducer as a waveguide of infinite cross section. A scattering matrix having both discrete and continuous indices, which correspond respectively to the mode in the ordinary waveguide and the continuum of modes in the waveguide of infinite cross section, is required. The expression of reciprocity, which seems to be of interest in itself in the class of problems considered, is established.

For the purpose of the present work, the scattering: matrices of the radiating-receiving systems involved are considered known; the determination of the necessary data for a practical application is considered to be an independent problem, experimental or possibly theoretical. (Fortunately in the practical cases under consideration, scattering by the radiatingreceiving systems involved is of minor importance, and it thus appears that the necessary data can be obtained from suitable measurements directly or indirectly determining the far-field radiation characteristics. Such measurements would yield the necessary information about the underlying diffraction problem, however complicated it might be.)

In section 3 the scattering matrix formalism is extended and applied to the calculation of the received signal in the basic arrangements described above. The general problem involving multiple reflections is soluble formally (in the sense that the problem is reducible to quadratures) in the form of a series of integrals, which stem from the LiouvilleNeumann series solution of a system of integral equations. Formulas are derived for models of the microwave Michelson and Fabry-Perot interferometers in which the radiating and receiving spectra are arbitrary. Finally, these formulas are illustrated by means of examples obtained by choosing specific and relatively simple analytical expressions for the spectra involved.

\section{Scattering Matrix Description of Radiat- ing-Receiving Systems}

A. Basic hypotheses. To the general description of the radiating-receiving systems given in section 1 we add the following details.

We choose a terminal surface $S_{0}$ in the waveguide and a supplementary surface $S_{0}^{\prime}$ (such that $S_{0}+S_{0}^{\prime}$ forms a closed surface) coinciding with the shielding around source, receiver, etc. (See fig. 1.) We further choose a rectangular coordinate system Oxyz such that coordinate surface $z=0$, which we denote by $S_{1}$, will serve as a terminal surface on the space side of the waveguide-space transducer. As a convenient artifice we employ an infinite hemisphere $S_{\infty}$ lying in $z \leq 0$ and centered at $\mathrm{O}$. The interior -i.e., the domain of the electromagnetic field - of the transducer is the region $V$ bounded externally by $S_{1}+S_{\infty}$ and internally by $S_{0}+S_{0}^{\prime}$. In figure 1 the structure of the transducer considered lies in $V$; other structures or objects may also be in $V$ but no attempt is made to illustrate this possibility.

The whole of the space and structure within $V$ is counted simply as a (decidedly) nonhomogeneous medium which may also be dissipative and anisotropic. Linearity is assumed and is essential; reciprocity, in the sense that tensors describing the medium within $V$ are required to be symmetric, is assumed and is useful but not essential.

In the space transmission paths (i.e., in $z>0$ ) the medium is to be homogeneous, isotropic, and nondissipative, as well as linear. For the time being it is assumed that these properties hold for arbitrarily large $z$; the interposition of elements (such as a reflecting object) will be considered later. (The theory is not actually restricted to transmission media having the ideal characteristics listed, since, as will be seen, an arbitrary linear transmission medium is to be treated as a suitable interposed element.)

It is assumed that the electromagnetic field quantities vary harmonically with time $t$ at frequency $\omega /(2 \pi)$. We employ the usual complex electric and magnetic field vectors, $\mathbf{E}, \mathbf{H}$, which are functions of the position vector $\mathbf{r}$ of Oxyz, and omit the time dependent factor $\exp (-i \omega t)$.

It should be observed that in taking $S_{\infty}$ to be hemispherical it is tacitly assumed that the problem is not two-dimensional (by a "two-dimensional" problem is meant one in which all quantities are independent of one rectangular coordinate perpendicular to the z-direction). To avoid unduly complicating the discussion, two-dimensional problems are not considered in the main part of this paper. However, two of the examples at the end of the paper are two-dimensional. For convenience in discussing these and other two-dimensional cases, some of the more important or less obvious of the needed formulas are summarized in an appendix (sec. 4, B).

B. Representation of field on $\mathrm{S}_{0}$. It, as is assumed, only one mode (a propagated mode) is of importance 
in the waveguide in the neighborhood of $S_{0}$, then from waveguide theory it is known that the transverse components $\mathbf{E}_{t}, \mathbf{H}_{t}$ of $\mathbf{E}, \mathbf{H}$ on $\mathrm{S}_{0}$ may be written

$$
\left.\begin{array}{rl}
\mathbf{E}(\mathbf{r})_{t} & =\left(a_{0}+b_{0}\right) \mathbf{e}(\mathbf{r}), \\
\mathbf{H}(\mathbf{r})_{t} & =\left(a_{0}-b_{0}\right) \mathbf{h}(\mathbf{r}),
\end{array}\right\}\left(\mathbf{r} \text { on } S_{0}\right)
$$

where $\mathbf{e}, \mathbf{h}$ are real basis-fields for the mode involved. The basis-fields are subject to the impedance normalization

$$
\mathbf{h}(\mathbf{r})=\eta_{0} \mathbf{n} \times \mathbf{e}(\mathbf{r})
$$

and the power normalization

$$
\int_{S_{0}} \mathbf{e}(\mathbf{r}) \times \mathbf{h}(\mathbf{r}) \cdot \mathbf{n} d S=4 \pi^{2} \eta_{0},
$$

where $\mathbf{n}$ is the unit normal on $S_{0}$ drawn into $V$ and $\eta_{0}$ is the wave-admittance for the mode involved. These equations implicitly define the quantities $a_{0}, b_{0}$; it can be verified that $a_{0}, b_{0}$ so defined are respectively linear measures of the electric field of the incident and emergent traveling-wave components of the waveguide field at $S_{0}$. The net time-average power input $P_{0}$ at $S_{0}$ is given by

$$
P_{0}=\operatorname{Re} \int_{S_{0}} \frac{1}{2} \mathbf{E} \times \overline{\mathbf{H}} \cdot \mathbf{n} d S=2 \pi^{2} \eta_{0}\left(\left|a_{0}\right|^{2}-\left|b_{0}\right|^{2}\right),
$$

where Re denotes that the real part is to be taken and the superposed bar denotes the complex conjugate.

C. Representation of the field in the region $\mathrm{z} \geq 0$. As mentioned in the introduction, the electromagnetic field in the region, $z \geq 0$ is to be represented as a superposition of plane-wave solutions of Maxwell's equations. This type of representation is well known, at least in the case of solutions of the scalar wave equation [4]; the generalization to the vector electromagnetic field offers no particular formal difficulty.

The electromagnetic field in the region under consideration satisfies Maxwell's equations in the form

$$
\nabla \times \mathbf{E}=i \omega \mu \mathbf{H}, \nabla \times \mathbf{H}=-i \omega \epsilon \mathbf{E},
$$

where $\mu, \epsilon$ are constant real scalars representing respectively the permeability and the permittivity of the medium. (Rationalized MKS units are employed.) We derive our basis fields from the general plane wave

$$
\left.\begin{array}{r}
\mathbf{E}=\mathbf{T} \exp (i \mathbf{k} \cdot \mathbf{r}), \\
\mathbf{H}=(\omega \mu)^{-1} \mathbf{k} \times \mathbf{T} \exp (i \mathbf{k} \cdot \mathbf{r}),
\end{array}\right\}
$$

which is a solution of (5) for any propagation vector $\mathbf{k}$ such that $k^{2}=\omega^{2} \mu \epsilon$ and any vector $\mathbf{T}$ satisfying the transversality relation $\mathbf{k} \cdot \mathbf{T}=0$. (In spite of this occurrence of "transversality," in what follows the term "transverse" will always mean transverse with respect to the $z$-direction.)

The propagation vector will be regarded as a function of its transverse components $k_{x}, k_{y}$; the $z$-component is then

$$
k_{z}= \pm \gamma
$$

where $\gamma=\left(k^{2}-k_{x}^{2}-k_{y}^{2}\right)^{\frac{1}{2}}$. It will be convenient to denote the transverse part of the propagation vector by $\mathbf{K}$, so that $\mathbf{K}=k_{x} \mathbf{e}_{x}+k_{y} \mathbf{e}_{y}$ and

$$
\gamma=\left(k^{2}-K^{2}\right)^{\frac{1}{2}} .
$$

Since $k_{x}, k_{y}$ must be allowed to vary independently in the range $(-\infty, \infty)$, real and imaginary values of $\gamma$ will occur. $\gamma$ will be taken positive for $K^{2}<k^{2}$, positive imaginary for $K^{2}>k^{2}$. Superscripts " + " and "-" will be used when it is desired to indicate the choice of sign associated with $k_{z}$.

In virtue of the relation $\mathbf{k} \cdot \mathbf{T}=0$, (6) yields just two linearly independent fields, hence just two basis fields, for any given $\mathbf{k}$. The appropriate polarizations for the basis fields are those with the electric vectors parallel or perpendicular to the plane of $\mathbf{k}$ and $\mathbf{e}_{z}$, which is the plane of incidence for a ray incident on any plane $z=$ const. This choice of polarizations corresponds to the practice in electromagnetic theory in deriving the Fresnel equations, for example; it also corresponds to the resolution into "transverse magnetic" and "transverse electric" modes of waveguide theory.

In setting up the desired basis fields it is convenient to employ the transverse unit vectors

$$
\boldsymbol{\kappa}_{1}=\mathbf{K} / K, \boldsymbol{\kappa}_{2}=\mathbf{e}_{z} \times \boldsymbol{\kappa}_{1},
$$

which are respectively parallel and perpendicular to the plane of $\mathbf{k}$ and $\mathbf{e}_{z}$. (The notation is illustrated in fig. 4.) As a temporary abbreviation we put $u^{ \pm}=\exp \left(i \mathbf{k}^{ \pm} \cdot \mathbf{r}\right)$. For the " $E_{\|} "$ (or TM) components we take $\mathbf{T}=\boldsymbol{\kappa}_{1} \mp K \gamma^{-1} \mathbf{e}_{z}$ and obtain from (6)

$$
\left.\begin{array}{c}
\mathbf{E}_{1}^{ \pm}=\left[\boldsymbol{\kappa}_{1 \mp} K \gamma^{-1} \mathbf{e}_{z}\right] u^{ \pm}, \\
\mathbf{H}_{1}^{ \pm}= \pm \eta_{1} \mathbf{e}_{z} \times \boldsymbol{\kappa}_{1} u^{ \pm},
\end{array}\right\}
$$

where $\eta_{1}=\omega \epsilon / \gamma$. For the " $E_{\perp}$ " (or $T E$ ) components we take $\mathbf{T}=\boldsymbol{\kappa}_{2}$ and obtain from (6)

$$
\left.\begin{array}{c}
\mathbf{E}_{2}^{ \pm}=\boldsymbol{\kappa}_{2} u^{ \pm} \\
\mathbf{H}_{2}^{ \pm}=\left[ \pm \eta_{2} \mathbf{e}_{2} \times \boldsymbol{\kappa}_{2}+K(\omega \mu)^{-1} \mathbf{e}_{z}\right] u^{ \pm}
\end{array}\right\}
$$

where $\eta_{2}=\gamma /(\omega \mu)$. Among other similarities it may be observed that $\eta_{1}, \eta_{2}$ are "wave-admittances" that correspond exactly to the wave-admittances encountered in the theory of waveguides with discrete modes. The expressions in (9) and (10) are essen- 


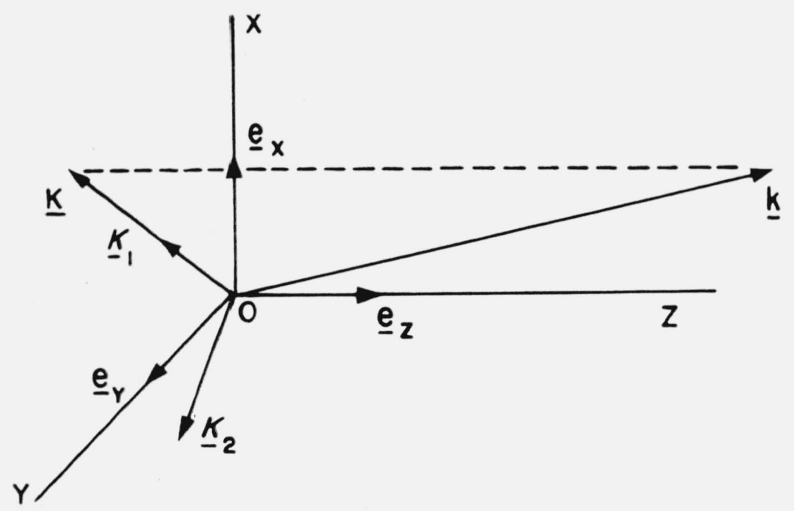

Figure 4. Illustrating $\mathbf{k}, \mathbf{K}, \kappa_{1}$, and $\kappa_{2}$.

tially the desired basis fields (z-components, included here, will be dropped later). The normalization and orthogonality properties of these fields are of course implicit in the expressions themselves.

We now introduce spectral density functions $b_{m}=b_{m}(\mathbf{K})$ and $a_{m}=a_{m}(\mathbf{K})$ for outgoing and incoming waves, respectively, and form the general superposition

$$
\left.\begin{array}{l}
\mathbf{E}(\mathbf{r})=\int \sum\left(b_{m} \mathbf{E}_{m}^{+}+a_{m} \mathbf{E}_{m}^{-}\right) d \mathbf{K} \\
\mathbf{H}(\mathbf{r})=\int \sum\left(b_{m} \mathbf{H}_{m}^{+}+a_{m} \mathbf{H}_{m}^{-}\right) d \mathbf{K}
\end{array}\right\}
$$

Here and subsequently in expressions of this type summation over the two values of the polarization index $m$ and integration over the domain of the two variables $k_{x}, k_{y}$ will be understood. The $\mathbf{E}, \mathbf{H}$ given by (11) will satisfy Maxwell's equations provided merely that the necessary differentiations can be taken under the integral sign. However, for our purposes, the $z$-components of the fields are redundant. By discarding the z-components we obtain the much more convenient and explicit expressions for the transverse field components

$$
\left.\begin{array}{c}
\mathbf{E}(\mathbf{r})_{t}=\int \sum\left(b_{m} e^{i \gamma z}+a_{m} e^{-i \gamma z}\right) \boldsymbol{\kappa}_{m} e^{i \mathbf{K} \cdot \mathbf{R}} d \mathbf{K}, \\
\mathbf{H}(\mathbf{r})_{t}=\int \sum\left(b_{m} e^{i \gamma z}-a_{m} e^{-i \gamma z}\right) \eta_{m} \mathbf{e}_{z} \times \boldsymbol{\kappa}_{m} e^{i \mathbf{K} \cdot \mathbf{R}} d \mathbf{K},
\end{array}\right\}
$$

where $\mathbf{R}$ denotes the transverse part of $\mathbf{r}$. These equations exhibit $\mathbf{E}_{t}, \mathbf{H}_{t}$ as two-dimensional Fourier transforms of the quantities multiplied by exp $(i \mathbf{K} \cdot \mathbf{R})$ in the respective integrands; from the inverse transformations one may obtain

$$
\left.\begin{array}{r}
b_{m}(\mathbf{K})=\frac{e^{-i \gamma z}}{8 \pi^{2}} \boldsymbol{\kappa}_{m} \cdot \int\left(\mathbf{E}+\eta_{m}^{-1} \mathbf{H} \times \mathbf{e}_{z}\right) e^{-i \mathbf{K} \cdot \mathbf{R}} d \mathbf{R}, \\
a_{m}(\mathbf{K})=\frac{e^{i \gamma z}}{8 \pi^{2}} \boldsymbol{\kappa}_{m} \cdot \int\left(\mathbf{E}-\eta_{m}^{-1} \mathbf{H} \times \mathbf{e}_{z}\right) e^{-i \mathbf{K} \cdot \mathbf{R}} d \mathbf{R} .
\end{array}\right\}
$$

Here and subsequently integrations with the differential symbol $d \mathbf{R}$ are to be taken over all values of $x$ and $y$ for some fixed $z \geq 0$. These equations enable one in principle to evaluate $a_{m}, b_{m}$ from a given distribution of $\mathbf{E}_{t}, \mathbf{H}_{t}$ on any transverse plane.

The case where there is no field incident on $S_{1}$ from the "right" [i.e., where $a_{m}(\mathbf{K}) \equiv 0$ ] is of particular interest in what follows, and we shall list several relations holding in this case. From (12) and (13)

$$
\begin{gathered}
\mathbf{E}(\mathbf{r})_{t}=\int \mathbf{B}(\mathbf{K}) e^{i \mathbf{k} \cdot \mathbf{r}} d \mathbf{K}, \\
\mathbf{B}(\mathbf{K})=(2 \pi)^{-2} e^{-i \gamma z} \int \mathbf{E}(\mathbf{r})_{t} e^{-i \mathbf{K} \cdot \mathbf{R}} d \mathbf{R}
\end{gathered}
$$

where $\mathbf{B}=\mathbf{B}(\mathbf{K})$ is the transverse vector $b_{1} \boldsymbol{\kappa}_{1}+b_{2} \boldsymbol{\kappa}_{2}$. The time-average power, $P_{r}$, radiated into the halfspace $z>0$ will also be of interest. This power is the same as the time-average power flux in the $+z$-direction across the surface $S_{1}$. Hence, from (12) [with $a_{m}(\mathbf{K}) \equiv 0$ ],

$P_{r}=\frac{1}{2} \operatorname{Re} \int \mathbf{E} \times \overline{\mathbf{H}} \cdot \mathbf{e}_{z} d \mathbf{R}=2 \pi^{2} \int_{K^{2}<k^{2}} \sum \eta_{m}(\mathbf{K})\left|b_{m}(\mathbf{K})\right|^{2} d \mathbf{K}$,

where, as indicated by the notation below the integral sign, evanescent waves are excluded from the integration. This equation is of a type known as Parseval's formulas; it may be derived formally with the aid of the rule that

$$
\int_{-\infty}^{\infty} e^{i\left(k_{x}-k_{x}^{\prime}\right) x} d x=2 \pi \delta\left(k_{x}-k_{x}^{\prime}\right)
$$

where $\delta$ denotes the so-called impulse or $\delta$-function [5].

D. Definition of scattering matrix. Having set up representations for the fields on the two terminal surfaces of the waveguide-space transducer under consideration, we are now in a position to consider the transducer as a whole. It may be assumed that a set of out-going wave-amplitudes $\left[b_{0}\right.$ and $\left.b_{m}(\mathbf{K})\right]$ will be determined by a set of incident wave-amplitudes $\left[a_{0}\right.$ and $\left.a_{m}(\mathbf{K})\right]$. In fact, since the electromagnetic system under consideration is by hypothesis a linear system, the relation between the set of out-going wave-amplitudes and the set of incident amplitudes must be a linear relation. We write this

$$
\begin{gathered}
b_{0}=S_{00} a_{0}+\int \sum S_{01}(m, \mathbf{K}) a_{m}(\mathbf{K}) d \mathbf{K} \\
b_{m}(\mathbf{K})=S_{10}(m, \mathbf{K}) a_{0}+\int \sum S_{11}(m, \mathbf{K} ; n, \mathbf{L}) a_{n}(\mathbf{L}) d \mathbf{L},
\end{gathered}
$$

thereby defining the scattering matrix for the transducer considered. Figure 1 may be helpful in fixing the significance of the quantities involved in (18). It is convenient and it seems appropriate to use the 
term "matrix" here even though one must think of rows and columns labeled both by discrete indices and by indices having continuous ranges. Evidently the functions $S_{01}, S_{10}$, and $S_{11}$ respectively embody the receiving properties, the radiating properties, and the (space-side) scattering properties of the transducer involved; $S_{00}$ is an ordinary waveguide reflection coefficient defined at the terminal surface $S_{0}$ and expressing the "antenna mismatch."

Equation (18) can be represented in terms of $S_{00}$ and linear functional operators $\hat{S}_{01}, \hat{S}_{10}$, and $\hat{S}_{11}$ such that

$$
\begin{aligned}
& b_{0}=S_{00} a_{0}+\hat{S}_{01} \hat{a}, \\
& \hat{b}=\hat{S}_{10} a_{0}+\hat{S}_{11} \hat{a},
\end{aligned}
$$

where $\hat{b}$ and $\hat{a}$ are understood as function vectors corresponding to the functions $b_{m}(\mathbf{K})$ and $a_{m}(\mathbf{K})$. This compact notation is used later primarily as a convenience in some of the more formal and general parts of the discussion.

E. Reciprocity. Radiating and receiving characteristics are related by the reciprocity condition, which here takes the form

$$
-\eta_{0} S_{01}(m, \mathbf{K})=\eta_{m}(\mathbf{K}) S_{10}(m,-\mathbf{K}) .
$$

Since this particular form of the condition appears to be new, a derivation is given below (appendix). The occurrence of the factors $\eta_{0}$ and $\eta_{m}(\mathbf{K})$ in (20) can be regarded as a consequence of the particular normalizations adopted in setting up the basis fields. (The same type of relationship holds for the elements of the scattering matrix of an ordinary waveguide junction with discrete modes [6].) The occurrence of the argument $-\mathbf{K}$ in one side of (20) means that the equation relates radiating and receiving characteristics in the line of a given propagation vector $\mathbf{k}$ (if a radiated wave has propagation vector $\mathbf{k}$, the received wave in the same line has the propagation vector $-\mathbf{k}$ ).

The plane-wave into plane-wave scattering function $S_{11}$ is also subject to reciprocity; the relation is

$\eta_{m}(\mathbf{K}) S_{11}(m,-\mathbf{K} ; n, \mathbf{L})=\eta_{n}(\mathbf{L}) S_{11}(n,-\mathbf{L} ; m, \mathbf{K})$.

Comments similar to those following (20) apply here also. This relation is not used in the present paper but it may well be of interest in other diffraction problems.

F. Determination of scattering matrix. Although the basic viewpoint of this paper is that $S_{00}, S_{01}$, etc., are to be considered known, it seems well to take some note of the problem of determining these quantities from empirical data in the arrangements of particular interest.

Concerning $S_{00}$ and $S_{11}$ there is not much to be said. $S_{00}$ is not only relatively easily measurable but also experimentally controllable (by means of tuning elements), whereas in general the same is not at all true of $S_{11}$. However, the desired operating condition that $S_{11}$ be effectively negligible is ap- proximately attainable and to some extent subject to experimental verification (by observation of the effects of multiple reflections).

Concerning $S_{01}$ and $S_{10}$ we first note: (1) Either one of these functions may be determined readily from the other with the aid of the reciprocity relation; (2) in the present context these functions need be evaluated only for $K^{2}<k^{2}$, the effects of evanescent waves being avoided by keeping $d>>\lambda$ (this is not inconsistent with Fresnel-region operation, cf sec. 1).

A direct approach to the determination of $S_{01}$ is implied by the definition (18): $S_{01}$ represents the received signal $b_{0}$ as a function of the direction and the polarization of incident plane waves of suitably normalized amplitudes.

According to (18), $S_{10}(m, \mathbf{K})$ represents the transverse components of the vector spectrum of outgoing waves under the conditions $a_{m}(\mathbf{K}) \equiv 0$ and $a_{0}=1$. If we define the transverse vectorial spectrum

$$
\mathbf{S}_{10}(\mathbf{K})=S_{10}(1, \mathbf{K}) \boldsymbol{\kappa}_{1}+S_{10}(2, \mathbf{K}) \boldsymbol{\kappa}_{2}
$$

then, from (15),

$$
\mathbf{S}_{10}(\mathbf{K})=\frac{1}{4 \pi^{2} a_{0}} \int \mathbf{E}_{t}(\mathbf{R}) e^{-i \mathbf{K} \cdot \mathbf{R}} d \mathbf{R} ;
$$

that is, $\mathbf{S}_{10}$ may be represented as the Fourier transform of the transverse components of the electric field obtaining on the reference plane in the absence of incident waves, normalized to unit $a_{0}$. This is, of course, essentially a vector form of the well-known relation between "aperture" distribution and spectrum.

The vectorial spectrum $\mathbf{S}_{10}(\mathbf{K})$ is also closely related to the far electric field by a well-known type of relation. Under certain restrictions $\mathbf{E}(\mathbf{r})_{t}$ has for large $r$ the asymptotic form [7]

$$
\mathbf{E}(\mathbf{r})_{t, \text { asymp }}=-2 \pi i k \cos \theta \mathbf{B}(\mathbf{R} k / r) e^{i k r} / r ;
$$

the angle $\theta$ introduced here is the polar angle of $\mathbf{r}$ relative to the $z$-axis. By rewriting this equation and dividing by $a_{0}$ we obtain a formula for $\mathbf{S}_{10}(\mathbf{K})$ in terms of the asymptotic form of $\mathbf{E}_{t}$ :

$$
\mathbf{S}_{10}(\mathbf{K})=i\left(2 \pi a_{0}\right)^{-1} \gamma^{-1} r e^{-i k r} \mathbf{E}(\mathbf{k} r / k)_{t, \text { asymp }},
$$

for $K^{2}<k^{2}$.

Finally we note that the familiar "power radiation pattern" or "polar diagram" of antenna theory, defined as radiated power per unit solid angle as a function of direction, is given by

$$
p=2(\boldsymbol{\epsilon} / \mu)^{\frac{1}{2}}(\pi k \cos \theta)^{2} \mathbf{b} \cdot \overline{\mathbf{b}},
$$

where $\mathbf{b}=\mathbf{B}+b_{z} \mathbf{e}_{z}$ and $b_{z}=-\mathbf{B} \cdot \mathbf{K} / \gamma \quad$ (b is the complete vectorial angular spectrum, including the $z$-component). Clearly this equation is not sufficient by itself to determine $\mathbf{S}_{10}(\mathbf{K})$; polarization and phase information is required in addition. 


\section{Applications}

A. Reflection systems. One obtains a form of the first basic arrangement described in section 1 by placing an infinite plane reflecting surface "in front of" a radiating-receiving system of the type considered in section 2. This represents a problem of intermediate complexity, from which the basic equations for the Michelson arrangement may be obtained by specialization. If the reflecting surface is at $z=d$ and has reflection coefficient $\rho(m, \mathbf{K})$, then, transforming the reflection coefficient to the plane $z=0$, we have

$$
a_{m}(\mathbf{K})=\rho(m, \mathbf{K}) e^{2 i \gamma(\mathbf{K}) d} \boldsymbol{b}_{m}(\mathbf{K})
$$

(the dependence of $\gamma$ on its arguments is now indicated explicitly). Upon substituting (27) into (18) one obtains

$$
b_{0}=S_{00} a_{0}+\int \sum S_{01}(m, \mathbf{K}) \rho(m, \mathbf{K}) e^{2 i \gamma(\mathbf{K}) d} b_{m}(\mathbf{K}) d \mathbf{K},
$$

$$
\begin{aligned}
b_{m}(\mathbf{K}) & =S_{10}(m, \mathbf{K}) a_{0} \\
& +\int \sum S_{11}(m, \mathbf{K} ; n, \mathbf{L}) \rho(n, \mathbf{L}) e^{2 i \gamma(\mathbf{L}) d} b_{n}(\mathbf{L}) d \mathbf{L} .
\end{aligned}
$$

The last line represents two simultaneous, inhomogeneous, linear integral equations for the determination of $b_{m}(\mathbf{K})$ ( $a_{0}$ being prescribed). The LiouvilleNeumann series solution of these equations may be obtained by a process of successive approximations. For the first approximation one takes

$$
b_{m}^{(1)}(\mathbf{K})=S_{10}(m, \mathbf{K}) a_{0} ;
$$

the second approximation is obtained by substituting the first into the right-hand side of (28b),

$$
\begin{aligned}
b_{m}^{(2)}(\mathbf{K})= & S_{10}(m, \mathbf{K}) a_{0} \\
& +a_{0} \int \sum S_{11}(m, \mathbf{K} ; n, \mathbf{L}) \rho(n, \mathbf{L}) e^{2 i \gamma(\mathbf{L}) d} S_{10}(n, \mathbf{L}) d \mathbf{L} ;
\end{aligned}
$$

and so on: $b_{m}^{(n)}(\mathbf{K})$ accounts for the first $n$ reflections at the reflecting surface. Once $b_{m}(\mathbf{K})$ is obtained, approximately or otherwise, it is to be substituted into (28a), thus determining the received waveamplitude $b_{0}$ in the waveguide at $S_{0}$.

Useful approximate equations describing the behavior of the Michelson instrument may now be obtained. The appropriate conditions are (1) that the effects of multiple reflections be negligible and (2) that the reflecting surface at $z=d$ be a mirror, for which we may put $\rho=-1$. The first condition means that (29) is already a good approximation for $b_{m}(\mathbf{K})$; this substituted into (28a) yields

$$
\frac{b_{0}}{a_{0}}=S_{00}-\int \sum S_{01}(m, \mathbf{K}) e^{2 i \gamma(\mathbf{K}) d} S_{10}(m, \mathbf{K}) d \mathbf{K} .
$$

The second term on the right will be called the "reflection integral" and denoted by $\Phi(d)$. Either $S_{01}$ or $S_{10}$ may be eliminated by means of the reciprocity condition (20); for the purposes of the present discussion it seems preferable to eliminate $S_{01}$. We then have

$\Phi(\mathrm{d})=\eta_{0}^{-1} \int \sum \eta_{m}(\mathbf{K}) S_{10}(m, \mathbf{K}) S_{10}(m,-\mathbf{K}) e^{2 i \gamma(\mathbf{K}) d} d \mathbf{K}$.

This is the main equation for the Michelson. It will be illustrated below by means of examples obtained by choosing specific mathematical expressions for $S_{10}(m, \mathbf{K})$.

Problems involving reflecting or scattering objects other than an effectively infinite reflecting surface are important not only in microwave interferometry but also in other fields. Consequently the following formulation of the general case where the plane reflecting surface considered above is replaced by an arbitrary reflecting object is of interest. Let the general reflecting object be characterized by means of a scattering function $R(m, \mathbf{K} ; n, \mathbf{L})$ defined with $z=0$ as terminal surface. Instead of (27) we now have the linear transformation

$$
a_{m}(\mathbf{K})=\int \sum R(m, \mathbf{K} ; n, \mathbf{L}) b_{n}(\mathbf{L}) d \mathbf{L}
$$

as boundary condition. In operator notation (33) is written

$$
\hat{a}=\hat{K} \hat{o}
$$

and the equations corresponding to (28) are

$$
\begin{aligned}
& b_{0}=S_{00} a_{0}+\hat{S}_{01} \hat{R} \hat{b}, \\
& \hat{b}=\hat{S}_{10} a_{0}+\hat{S}_{11} \hat{R} \hat{b} .
\end{aligned}
$$

The Liouville-Neumann series solution of (34b) may be written

where

$$
\hat{b}=\hat{S}_{10} \hat{a}_{0}+\hat{L} \hat{S}_{10} \hat{a}_{0}
$$

$$
\hat{L}=\sum_{p=1}^{\infty}\left(\hat{S}_{11} \hat{K}\right)^{p}
$$

is the operator corresponding to what is called the "resolvent kernel" in the theory of integral equations. Finally, for $b_{0}$ we obtain

$$
b_{0}=S_{00} a_{0}+\hat{S}_{01} \hat{R} \hat{S}_{10} a_{0}+\hat{S}_{01} \hat{R} \hat{L} \hat{S}_{10} a_{0}
$$

In this form the last term (specifically the operator $\hat{L})$ accounts for multiple reflections.

An instance of (37) is the case of a large but finite mirror in the Michelson interferometer, which has been considered using an approximate expression for $\hat{R}$ and neglecting multiple reflections [8]. 
B. Transmission systems; Fabry-Perot interferometer. We now consider a general transmission system consisting of a radiating system and a receiving system with an arbitrary (electromagnetically linear) intervening structure or medium. For the active terminal the pertinent descriptive equation is (19b), which for convenience is repeated here:

$$
\hat{b}=\hat{S}_{10} a_{0}+\hat{S}_{11} \hat{a} .
$$

This equation is understood to be set up with reference to a coordinate system Oxyz and spaceside reference plane $z=0$, as detailed in section 2 . Using primes to distinguish quantities associated with the passive terminal, we may write

$$
\begin{aligned}
& b_{0}^{\prime}=\hat{S}_{01}^{\prime} \hat{a}^{\prime}, \\
& \hat{b}^{\prime}=\hat{S}_{11} \hat{a}^{\prime},
\end{aligned}
$$

as the equations corresponding to (19). For these equations the space-side reference plane is $z=d$ in the above-mentioned coordinate system and the general arrangement is shown in figure 3 . Next, let the structure and/or medium between the terminals be described by a set of linear operators $\hat{T}_{i j}$, defined with respect to $z=0$ and $z=d$ as reference planes, such that

$$
\begin{array}{r}
\hat{a}=\hat{T}_{11} \hat{b}+\hat{T}_{12} \hat{b}^{\prime}, \\
\hat{a}^{\prime}=\hat{T}_{21} \hat{b}+\hat{T}_{22} \hat{b}^{\prime} .
\end{array}
$$

(The Fabry-Perot interferometer considered below will furnish an example of these equations. It will be a very special example, however, since specular reflection and transmission will be assumed, so that the operators $\hat{T}_{i j}$ will be diagonal and (40) is then reducible to a family of ordinary equations.)

A method of solving the problem described in (19), (39), and (40) may be indicated as follows. The result of eliminating $\hat{b}$ and $\hat{b}^{\prime}$ from $(19 \mathrm{~b}),(39 \mathrm{~b})$, and (40) may be put in the form

$$
\left[\begin{array}{l}
\hat{a} \\
\hat{a}^{\prime}
\end{array}\right]=\left[\begin{array}{c}
\hat{T}_{11} \hat{S}_{10} a_{0} \\
\hat{T}_{21} \hat{S}_{10} a_{0}
\end{array}\right]+\left[\begin{array}{ll}
\hat{T}_{11} \hat{S}_{11} & \hat{T}_{12} \hat{S}_{11}^{\prime} \\
\hat{T}_{21} \hat{S}_{11} & \hat{T}_{22} \hat{S}_{11}^{\prime}
\end{array}\right]\left[\begin{array}{c}
\hat{a} \\
\hat{a}^{\prime}
\end{array}\right] .
$$

As this form suggests, these equations may be solved for $\hat{a}$ and $\hat{a}^{\prime}$ by a process of successive approximations similar to that used above. The received wave amplitude $b_{0}^{\prime}$ is directly determinable from (39a) as soon as $\hat{a}^{\prime}$ is known. ( $b_{0}$ is also directly determinable.)

To pass to the consideration of a highly simplified model of the Fabry-Perot interferometer, we first assume that $\hat{S}_{11}$ and $\hat{S}_{11}^{\prime}$ are effectively so small that reflections at the terminals of the system may be neglected; that is, we assume that the second term on the right in (41) may be neglected. We then have the explicit expression

$$
\hat{a}^{\prime}=\hat{T}_{21} \hat{S}_{10} a_{0}
$$

and consequently for the received wave-amplitude we have

$$
b_{0}^{\prime}=\hat{S}_{01}^{\prime} \hat{T}_{21} \hat{S}_{10} a_{0}
$$

Next, we assume that the structure described by the $\hat{T}_{i j}$ is a Fabry-Perot "etalon", consisting of a pair of elements corresponding to the two plates of an optical Fabry-Perot interferometer. Such elements might be, for example, perforated metal sheets or stacked quartz plates [8]. We assume that each element is symmetric with respect to the z-direction and characterizable by means of a (specular) reflection coefficient $\rho(m, \mathbf{K})$ and a (specular) transmission coefficient $\tau(m, \mathbf{K})$ defined at the symmetry plane of the element as reference plane. It is not assumed that the elements are lossless; $\rho$ and $\tau$ are subject merely to realizability conditions for passive elements. We let the two elements be located so that their reference planes coincide with $z=0$ and with $z=d$, respectively. It may be noted that so locating the elements implies no real loss of generality, since the planes $z=0$ and $z=d$ are arbitrarily located with respect to the physical arrangements with which they are respectively associated. From the symmetry of the etalon as a whole with respect to $z=d / 2$ and the fact that the $\hat{T}_{i j}$ must be diagonal we have

$$
\begin{aligned}
& T_{11}=T_{22}=\delta_{m n} \delta(\mathbf{K}-\mathbf{L}) t_{11}(m, \mathbf{K}) \\
& T_{21}=T_{12}=\delta_{m n} \delta(\mathbf{K}-\mathbf{L}) t_{21}(m, \mathbf{K}),
\end{aligned}
$$

where $\delta(\mathbf{K}-\mathbf{L})$ means $\delta\left(k_{x}-l_{x}\right) \delta\left(k_{y}-l_{y}\right)$ and

$$
\begin{gathered}
t_{11}=\rho \frac{1+\left(\tau^{2}-\rho^{2}\right) e^{2 i \gamma d}}{1-\rho^{2} e^{2 i \gamma d}}, \\
t_{21}=\frac{\tau^{2} e^{i \gamma d}}{1-\rho^{2} e^{2 i \gamma a}},
\end{gathered}
$$

as may be found by ordinary methods. In these expressions $\gamma, \rho$, and $\tau$ in general depend upon $\mathbf{K}$. When evaluated for $\mathbf{K}=0$ and simplified somewhat as they may be for lossless elements, the expressions reduce to ones frequently used in discussions of the optical Fabry-Perot interferometer.

Using (44b) and writing out (43) we obtain

$$
b_{0}^{\prime}=a_{0} \int \sum S_{01}^{\prime}(m, \mathbf{K}) t_{21}(m, \mathbf{K}) S_{10}(m, \mathbf{K}) d \mathbf{K} ;
$$

finally, inserting (45b) and defining $\Psi(d)=b_{0}^{\prime} / a_{0}$,

$$
\Psi(d)=\int \sum \frac{S_{01}^{\prime}(m, \mathbf{K}) \tau^{2}(m, \mathbf{K}) e^{i \gamma(\mathbf{K}) d} S_{10}(m, \mathbf{K}) d \mathbf{K}}{1-\rho^{2}(m, \mathbf{K}) e^{2 i \gamma(\mathbf{K}) d}} .
$$


This is the "transmission integral" for a FabryPerot interferometer. An example of the analytical evaluation of (46) will be given below, assuming constant $\rho$ and $\tau$ and choosing very simple expressions for $S_{01}$ and $S_{10}$. (It may be observed that in regarding (46) as a function of $d$, it is implicit that the terminal apparatuses remain fixed relative to the reference planes with which they are respectively associated.)

The examples that follow have been chosen to illustrate various features of the theory and of the instruments considered. Inasmuch as the examples are rather hypothetical in nature and mainly illustrative, no thorough or rigorous discussions are attempted.

C. Examples, Michelson case.

C.1. Dipole. The following example seems wellsuited to illustrate the theory, inasmuch as it involves both TE and TM field-components in a fairly complicated way, the integrals involved can be evaluated, and the form of the answer can be anticipated. In this example the radiated field is assumed to be identical to that of an elementary electric dipole of moment $\mathbf{p}$ located at $\mathrm{O}$.

To find the angular spectrum of the electric field we may proceed as follows. The appropriate Hertz potential is [9]

$$
\mathbf{\Pi}=(4 \pi \epsilon)^{-1} \mathbf{p} e^{i k r} / r ;
$$

the representation of the spherical wave $\exp (i k r) / r$ in terms of plane waves is [10], for $z>0$,

$$
e^{i k r} / r=-(2 \pi i)^{-1} \int \gamma^{-1} e^{i \mathbf{k} \cdot \mathbf{r}} d \mathbf{K}
$$

where $\mathbf{k}=\mathbf{k}^{+}$is understood. Since $\mathbf{E}=\nabla \times \nabla \times \mathbf{I}$ we have

$$
\mathbf{E}=C \int \mathbf{k} \times(\mathbf{k} \times \mathbf{p}) \gamma^{-1} e^{i \mathbf{k} \cdot \mathbf{r}} d \mathbf{K},
$$

where $C=\left(8 \pi^{2} \epsilon i\right)^{-1}$. Hence the complete vectorial spectrum is $\mathbf{b}=C \mathbf{k} \times(\mathbf{k} \times \mathbf{p}) \gamma^{-1}$. This result holds for alln ; immediate confirmation for $K^{2}<k^{2}$ may be obtained from (25) using the asymptotic form

$$
-k^{2}(4 \pi \boldsymbol{\epsilon})^{-1} \mathbf{r} \times(\mathbf{r} \times \mathbf{p}) e^{i k r} / r^{3}
$$

for $\mathbf{E}$ [9].

According to the definition of $S_{10}(m, \mathbf{K})$, we have in this example

$$
S_{10}(m, \mathbf{K})=\left(C / a_{0}\right) \boldsymbol{\kappa}_{m} \cdot[\mathbf{k} \times(\mathbf{k} \times \mathbf{p})] \gamma^{-1}
$$

for $m=1,2$. These spectral components are to be substituted into the reflection integral (32) for the Michelson instrument. The coefficient of $\exp (2 i \gamma d)$ in the integrand of (32), after some vector-algebraic labor, is found in the present instance to be expressible in the form

$$
-\omega \epsilon\left(C / a_{0}\right)^{2} \mathbf{p} \cdot\left[\mathbf{k} \times\left(\mathbf{k} \times \mathbf{p}^{\prime}\right)\right] \gamma^{-1},
$$

where $\mathbf{p}^{\prime}$ is the negative mirror image of $\mathbf{p}$, with components $\left(-p_{x},-p_{y}, p_{z}\right)$. Thus for (32) we have

$\Phi(d)=-\omega \epsilon \eta_{0}^{-1}\left(C / a_{0}\right)^{2} \mathbf{p} \cdot \int \mathbf{k} \times\left(\mathbf{k} \times \mathbf{p}^{\prime}\right) \gamma^{-1} e^{2 i \gamma d} d \mathbf{K}$.

Comparing this expression with (47) it is seen that

$$
\Phi(d)=-\frac{C \omega \epsilon}{\eta_{0} a_{0}^{2}} \mathbf{p} \cdot \mathbf{E}^{\prime}(0,0,2 d)
$$

where $\mathbf{E}^{\prime}$ is the electric field of a dipole of moment $\mathbf{p}^{\prime}$ located at $\mathrm{O}$, and $\mathbf{E}^{\prime}$ is evaluated at the image of $\mathrm{O}$ in the reflecting surface of the instrument. This result is indeed of a form that might be anticipated.

If for simplicity we take $\mathbf{p}$ to be transverse, (50) becomes more explicitly [9],

$$
\Phi(d)=C_{1}\left[(2 i k d)^{-3}-(2 i k d)^{-2}+(2 i k d)^{-1}\right] e^{2 i k d},
$$

where $C_{1}$ is independent of $d$. It is clear that the result in this example is not physically meaningful as $k d \rightarrow 0$, for the magnitude of $\Phi$ can not properly exceed unity. This defect is attributable to the neglect of scattering or re-radiation by the dipole; a plausible extension of the theory of this example taking scattering into account gives results qualitatively well-behaved for all values of $k d$.

C.2 Two-dimensional Gaussian.-To provide a reasonably simple analytical illustration of the behavior of a Michelson instrument operated in the Fresnel region, we consider a two-dimensional case with the pure TM "Gaussian" spectrum

$$
\begin{aligned}
& b_{1}\left(k_{x}\right)=A e^{-a^{2} k_{x}^{2} / 2}, \\
& b_{2}\left(k_{x}\right)=0 .
\end{aligned}
$$

The subscripts 1, 2 here refer to $\mathbf{e}_{\mathbf{x}}, \mathbf{e}_{\mathbf{y}}$, respectively, and $A$ is an arbitrary amplitude. The corresponding distribution of $\mathbf{E}_{t}$ on the reference plane $z=0$ is also Gaussian,

$$
\begin{aligned}
& E_{x}=\text { Const. } e^{-x^{2} /\left(2 a^{2}\right)}, \\
& E_{y}=0,
\end{aligned}
$$

as follows from $\left(14_{2}\right)$ (see appendix, B). The parameter $a$ is, in a well-known manner, a measure of the sharpness of the angular spectrum and a measure of the width of the distribution of $\mathbf{E}_{t}$.

In this example it will be interesting to determine the absolute magnitude of $\Phi$ explicitly. We need the relation between $\left|a_{0}\right|$ and $|A|$ and we obtain this from a consideration of energy balance under freespace radiation conditions. If the fraction $h$ of the net input power at $S_{0}$ is radiated, we have

$$
\pi \eta_{0}\left|a_{0}\right|^{2}\left(1-\left|S_{00}\right|^{2}\right) h=\pi \int_{-k}^{k} \sum \eta_{m}\left|b_{m}\left(k_{x}\right)\right|^{2} d k_{x},
$$


where the left- and the right-hand sides of this equation come from $\left(4_{2}\right)$ and $\left(16_{2}\right)$, respectively.

The appropriate two-dimensional form of (32) is

$\Phi(d)=-\eta_{0}^{-1} \int \sum \eta_{m}\left(k_{x}\right) S_{10}\left(m, k_{x}\right) S_{10}\left(m,-k_{x}\right) e^{2 i \gamma\left(k_{x}\right) d} d k_{x}$,

where we still have $\eta_{1}=\omega \epsilon \gamma^{-1}$ and $\eta_{2}=\gamma(\omega \mu)^{-1}$. In the present case this becomes

$$
\Phi(d)=-\frac{A^{2} \omega \epsilon}{\eta_{0} a_{0}^{2}} \int \gamma^{-1} \exp \left(-a^{2} k_{x}^{2}+2 i \gamma d\right) d k_{x} .
$$

Letting $\phi$ denote the phase of $A / a_{0}$ and using (52) to eliminate $\left|A / a_{0}\right|$ from the last equation, one obtains

$\Phi(d)=-e^{2 i \phi}\left(1-\left|S_{00}\right|^{2}\right) h \frac{\int \gamma^{-1} \exp \left(-a^{2} k_{x}^{2}+2 i \gamma d\right) d k_{x}}{\int_{-k}^{k} \gamma^{-1} \exp \left(-a^{2} k_{x}^{2}\right) d k_{x}}$.

If $k a>>1$, this expression yields $\Phi(0) \cong-e^{2 i \phi}$ $\left(1-\left|S_{00}\right|^{2}\right) h$-a quantity that may approximate unity in magnitude.

Since we are interested in Fresnel-region behavior, an asymptotic expansion of the numerator of (54) in terms of inverse powers of $a$ is appropriate. This asymptotic expansion may be found with the aid of Watson's lemma, as given by Jeffreys and Jeffreys [11]. One finds

$$
\begin{aligned}
\Phi(d)=C_{1} e^{2 i k d}[1+ & \frac{1-2 i k d}{4(k a)^{2}} \\
& \left.+\frac{9-18 i k d-12(k d)^{2}}{32(k a)^{4}}+\cdots\right],
\end{aligned}
$$

where $C_{1}$ does not depend upon $d$. For this expression to yield a good approximation it is necessary that $k a>>1$ and that $k a^{2}>>d$ (these inequalities are equivalent to those given in section 1 in the description of Fresnel-region operation). To the second order in $k a$,

$$
\arg \Phi(d) \cong 2 k d\left[1-(2 k a)^{-2}\right] .
$$

Thus the "diffraction correction" to the phase can be expressed in terms of a small increase in effective wavelength, which in this approximation and in this example is independent of $d$.

It is of some interest to evaluate this result for values of $k$ and $a$ that might be considered typical of optical cases, even though the formula does not apply, or at least does not apply directly, to any optical instrument. If one takes $\lambda=5000 \mathrm{~A}$ and $a=5$ $\mathrm{cm}$, the fractional increase in effective wavelength given by the formula is approximately $6 \times 10^{-13}$.

C.3 TE $E_{10}$ aperture-distribution. A somewhat more realistic - and much more intractable-example of Fresnel region behavior is afforded by the assumption of a $\mathrm{TE}_{10}$-mode distribution in a square aperture in a conducting screen. This example is suggested by an experimental arrangement in which a square horn-lens radiator is fed by a taper from rectangular waveguide supporting the $\mathrm{TE}_{10}$ mode [8]. If the aperture is bounded by $|x|=a / 2$ and $|y|=a / 2$ in the plane $z=0$, we may take

$$
\mathbf{E}_{\iota}=\cos (\pi x / a) \mathbf{e}_{y}
$$

in the aperture and $\mathbf{E}_{t}=0$ elsewhere in the plane. From $(22)$ and (23) it follows that

$$
\begin{aligned}
& S_{10}(1, \mathbf{K})=k_{y} f(\mathbf{K}) /|K|, \\
& S_{10}(2, \mathbf{K})=k_{x} f(\mathbf{K}) /|K|,
\end{aligned}
$$

where

$$
f(\mathbf{K})=C \frac{\cos \left(k_{x} a / 2\right)}{(\pi / a)^{2}-k_{x}^{2}} \cdot \frac{\sin \left(k_{y} a / 2\right)}{k_{y}} .
$$

(Here and subsequently unimportant constant factors are denoted $C, C^{\prime}$, etc., without explicit definition in each case.) Hence (32) becomes

$$
\Phi(d)=C^{\prime} \int\left(\frac{\omega \epsilon}{\gamma} k_{y}^{2}+\frac{\gamma}{\omega \mu} k_{x}^{2}\right) \frac{[f(\mathbf{K})]^{2}}{K^{2}} e^{2 i \gamma d} d \mathbf{K} .
$$

This integral has been evaluated numerically. The numerical analysis and the programming required for this difficult task were performed by Paul F. Wacker and William W. Longley, Jr. Some of the results are shown in table 1 , wherein the quantity labeled $\Delta d$ is calculated in accordance with the definition

$$
\Delta d=(2 k)^{-1}[\arg \Phi(d)-\arg \Phi(0)]-d .
$$

TABLE 1

\begin{tabular}{c|r|r|r}
\hline$\lambda$ & \multicolumn{1}{c|}{$a$} & \multicolumn{1}{c|}{$d$} & \multicolumn{1}{c}{$\Delta d$} \\
\cline { 1 - 1 }$c m$ & \multicolumn{1}{c|}{$c m$} & \multicolumn{1}{c}{$m$} & \multicolumn{1}{c}{ Microns } \\
0.6278 & 60 & 2 & -56.96 \\
.6278 & 60 & 10 & -193.80 \\
.6278 & 30 & 2 & -155.93 \\
.6278 & 30 & 10 & -503.28 \\
.1 & 60 & 2 & -2.32 \\
.1 & 60 & 10 & -7.56 \\
\hline
\end{tabular}


The fact that this quantity is negative corresponds to the general fact that the effective wavelength tends to be greater than the free-space wavelength, so that the observed phase increases with $d$ more slowly than $2 k d$.

D. Fabry-Perot with line source. In this example the radiated field of the radiating system is taken to be identical to that of a simple line current coinciding with the $y$-axis, and it is assumed that the receiving system is the same as the radiating system. These assumptions yield a two-dimensional, pure TE problem in which the free-space radiation pattern has no Fresnel region. Although only the most readily obtainable results are given here, the example already affords an interesting illustration of the behavior of a Fabry-Perot instrument in the presence of a continuous angular spectrum.

The assumed field being pure TE, we certainly have $S_{10}\left(1, k_{x}\right)=0$; and since $\mathbf{E}$ is asymptotically proportional to $r^{-1 / 2} \mathrm{e}^{i k r} \mathbf{e}_{y}$, it follows from $\left(25_{2}\right)$ that we may write $S_{10}\left(2, k_{x}\right)=\gamma^{-1}$, at least for $k_{x}^{2}<k^{2}$. We shall employ this expression for the whole range of $k_{x}$, choosing to justify this analytic continuation by the results to be obtained in a moment. Using the reciprocity relation $\left(20_{2}\right)$ we obtain for the receiving system $S_{01}^{\prime}\left(2, k_{x}\right)=\gamma(\omega \mu)^{-1} \eta_{0}^{-1} S_{10}^{\prime}\left(2,-k_{x}\right)=$ $\left(\omega \mu \eta_{0}\right)^{-1}$. Hence the transmission integral (46) becomes

$$
\Psi(d)=C \int_{-\infty}^{\infty} \frac{\tau^{2} e^{i \gamma d} \gamma^{-1}}{1-\rho^{2} e^{2 i \gamma d}} d k_{x}
$$

The integral diverges for $d=0$; this behavior is attributable to the implicit assumption of an enforced current in the radiating element (as in the dipole example above). If we assume $\tau$ and $\rho$ constants independent of $k_{x}, d>0$, and $|\rho|<1$, then (58) may be written

$$
\Psi(d)=C \tau^{2} \sum_{n=0}^{\infty} \rho^{2 n} \int_{-\infty}^{\infty} \gamma^{-1} e^{i(2 n+1) \gamma d} d k_{x} .
$$

By introducing a new variable of integration $\alpha$, such that $k \sin \alpha=-\gamma$, one may transform the integral to one of the standard representations of the Hankel function [12] of the first kind and order zero. Thus

$$
\Psi(d)=C^{\prime} \tau^{2} \sum_{n=0}^{\infty} \rho^{2 n} H_{0}^{(1)}[(2 n+1) k d] .
$$

At this point we note that for $\rho=0, \Psi(\mathrm{d})$ becomes proportional to $H_{0}^{(1)}(k d)$, as might be expected for free-space transmission between systems of the description considered.

For $|\rho|$ approaching unity, features peculiar to the Fabry-Perot may be expected to appear-and the series becomes very slowly convergent. A thorough discussion of (59) might be premature and will not be attempted here (one might wish to consider a more realistic model of the Fabry-Perot, for example). We shall assume $k d>>1$ and approximate the Hankel function by means of the first term of its asymp- totic expansion. Instead of (59) we then have

$$
\Psi(d) \cong C^{\prime \prime} \tau^{2} \rho^{-1}(k d)^{-\frac{1}{2}} F(\rho, k d),
$$

where

$$
F(\rho, k d)=\sum_{n=0}^{\infty}(2 n+1)^{-\frac{1}{2}}\left(\rho e^{i k d}\right)^{2 n+1} .
$$

In (60b) a phase angle in $\rho$ obviously is equivalent to an additive constant in $d$; having noted this, we assume the $\rho^{2}$ is real and positive.

For $\rho^{2}<1$, the series $(60 b)$ converges for all values of $d$ and $|F(\rho, k d)|$ has maxima for $k d=m \pi$, where $m$ is an integer (for $\rho^{2}=1$, the series still converges for $k d \neq m \pi$ but diverges infinitely at the points where $k d=m \pi)$. These maxima are certainly the principa] maxima and correspond to the passage of axial rays; it is not known whether there are subsidiary maxima.

Since $|\tau|^{2} \leq 1-|\rho|^{2}$ (the equality holding for a lossless etalon), $\tau$ and hence $\Psi$ must approach zero as $\rho^{2} \rightarrow 1$ except possibly at the values for $d$ for which the series diverges. An estimate of $F(\rho, m \pi)$ as $\rho^{2} \rightarrow 1$ indicates that $\Psi$ must approach zero at these points also. This decrease of transmission at the maxima as $\rho^{2} \rightarrow 1$ differs from the result given by the optical formula (45b) and may be surprising. It can be explained in terms of increasing selectivity for axial rays, such that a decreasing portion of the incident spectrum, and hence decreasing power, is transmitted.

Finally it may be observed that $F(\rho, k d)$ is a periodic function of $d$, so that the factor $d^{-\frac{1}{2}}$ in (60a) gives the general trend of $\Psi$ with $d$. In the optical case, $\Psi$ itself would be periodic.

\section{Appendix}

A. Reciprocity relations. Let $\mathbf{E}^{\prime}, \mathbf{H}^{\prime}$, and $\mathbf{E}^{\prime \prime}, \mathbf{H}^{\prime \prime}$ denote any two electromagnetic fields (of the same frequency) that can exist in the interior of the waveguide-space transducer considered. In virtue of the hypotheses imposed in section 2, the Lorentz relation

$$
\nabla \cdot\left(\mathbf{E}^{\prime} \times \mathbf{H}^{\prime \prime}-\mathbf{E}^{\prime \prime} \times \mathbf{H}^{\prime}\right)=0
$$

will hold in $V[6]$. Therefore, using the divergence theorem, one has

$$
\int_{S_{0}+S_{0}^{\prime}+S_{1}+S_{\infty}}\left(\mathbf{E}^{\prime} \times \mathbf{H}^{\prime \prime}-\mathbf{E}^{\prime \prime} \times \mathbf{H}^{\prime}\right) \cdot \mathbf{n} d S=0,
$$

where $\mathbf{n}$ is the inward unit normal on the boundary of $V$ and the parts $S_{0}, S_{0}^{\prime}$, etc., making up the boundary of $V$ are as defined in section 2. Now, the contribution of the integral over $S_{0}^{\prime}$ vanishes, since the integrand vanishes there. By using the asymptotic form of the fields for large $r$, it can be shown that the $S_{\infty}$-integral vanishes for fields generated by any distribution of sources confined to a sphere of finite radius centered at $\mathrm{O}$. This restriction 
apparently would rule out excitation of the system by plane waves incident on $S_{1}$, which we wish to consider, but since we can imagine a plane wave approximated arbitrarily closely by a source of finite dimensions at a sufficiently large distance, there is no real limitation. Hence we may employ the relation

$$
\int_{S_{0}+S_{1}}\left(\mathbf{E}^{\prime} \times \mathbf{H}^{\prime \prime}-\mathbf{E}^{\prime \prime} \times \mathbf{H}^{\prime}\right) \cdot \mathbf{n} d S=0
$$

without explicit restrictions. If in this expression one replaces $\mathbf{E}^{\prime}, \mathbf{H}^{\prime}$ and $\mathbf{E}^{\prime \prime}, \mathbf{H}^{\prime \prime}$ by their representations on $S_{0}$ and $S_{1}$, equations (1) and (12), one finds after some analysis

$$
\begin{aligned}
& \eta_{0}\left(a_{0}^{\prime} b_{0}^{\prime \prime}-a_{0}^{\prime \prime} b_{0}^{\prime}\right)-\int \Sigma \eta_{m}(\mathbf{K}) {\left[a_{m}^{\prime}(\mathbf{K}) b_{m}^{\prime \prime}(-\mathbf{K})\right.} \\
&\left.-a_{m}^{\prime \prime}(\mathbf{K}) b_{m}^{\prime}(-\mathbf{K})\right] d \mathbf{K}=0 .
\end{aligned}
$$

In obtaining this result the use of the integral representation (17) of the $\delta$-function is helpful.

We now assume that $\mathbf{E}^{\prime}, \mathbf{H}^{\prime}$ and $\mathbf{E}^{\prime \prime}, \mathbf{H}^{\prime \prime}$ are the fields corresponding to excitation by the following particular sets of incident waves

$$
\begin{aligned}
a_{0}^{\prime} & =1, & a_{0}^{\prime \prime} & =0, \\
a_{m}^{\prime}(\mathbf{K}) & =0 ; & a_{m}^{\prime \prime}(\mathbf{K}) & =\delta_{m n} \delta(\mathbf{K}-\mathbf{L}) .
\end{aligned}
$$

From the scattering equations (18) we obtain

$$
b_{m}^{\prime}(\mathbf{K})=S_{10}(m, \mathbf{K}), \quad b_{0}^{\prime \prime}=S_{01}(n, \mathbf{L}) .
$$

Upon substituting all these quantities into (62) and observing that $\eta_{m}(\mathbf{L})=\eta_{m}(-\mathbf{L})$, one obtains the reciprocity relation (20) of the text.

In a very similar manner one can derive (21) of the text.

B. Two-dimensional formulation. For the discussion of two-dimensional cases many, if not most, of the formulas of the text require modification, and almost all the modifications may readily be obtained by reduction from formulas given in the text or by parallel development. Certain key formulas and minor subtleties are discussed here.

It is assumed that all quantities are independent of the transverse coordinates $y$ and $k_{y}$, so that these coordinates will be absent from all formulas.

Under the above assumption the unit vectors $\boldsymbol{\kappa}_{1}$, $\boldsymbol{\kappa}_{2}$ designating the "parallel" and "perpendicular" electric-field directions degenerate to $\boldsymbol{\kappa}_{1}=\mathbf{e}_{x} \operatorname{sgn} k_{x}$, $\boldsymbol{\kappa}_{2}=\mathbf{e}_{y} \operatorname{sgn} k_{x}$. The inconvenience of the sign reversal is avoided by adopting $\mathbf{e}_{x}, \mathbf{e}_{y}$ as the basis vectors for the two polarizations. Sums over the polarization index $m$ become sums over $x$ - and $y$ components. This change induces a few further sign changes, the key one being in the reciprocity relation (given below).
The two-dimensional forms of (14) and (15) may be written

$$
\begin{gathered}
\mathbf{E}(\mathbf{r})_{t}=\int \mathbf{B}\left(k_{x}\right) e^{i k_{x} x+i \gamma z} d k_{x}, \\
\mathbf{B}\left(k_{x}\right)=(2 \pi)^{-1} e^{-i \gamma z} \int \mathbf{E}(\mathbf{r})_{t} e^{-i k_{x} x} d x
\end{gathered}
$$

where $\quad \mathbf{r}=x \mathbf{e}_{x}+z \mathbf{e}_{z}, \quad \gamma^{2}=k^{2}-k_{x}^{2}$, and integrations with respect to $k_{x}$ and $x$ are understood to be taken over the range $(-\infty, \infty)$. (The numbering of these two equations indicates the scheme to be followed in this appendix.)

Power expressions such as (16) and (4) must be reinterpreted as power per unit length in the $y$-direction. Equation (16) becomes

$$
P_{r}=\pi \int_{k_{x}^{2}<k^{2}} \sum \eta_{m}\left|b_{m}\left(k_{x}\right)\right|^{2} d k_{x} .
$$

It is convenient to match the loss of a factor of $2 \pi$ between (16) and $\left(16_{2}\right)$ by renormalizing the basis fields at $S_{0}$ so that (4) becomes

$$
P_{0}=\pi \eta_{0}\left(\left|a_{0}\right|^{2}-\left|b_{0}\right|^{2}\right) \cdot
$$

With this renormalization the numerical factors in the reciprocity relation remain unchanged.

The reciprocity relation becomes

$$
\eta_{0} S_{01}\left(m, k_{x}\right)=\eta_{m} S_{10}\left(m,-k_{x}\right) \cdot
$$

This is probably best established by a derivation parallel to that used for (20). The disappearance of a minus sign between the members of $\left(20_{2}\right)$ as compared with (20) is due to the adoption of $\mathbf{e}_{x}$ and $\mathbf{e}_{y}$ as basis vectors for the two polarizations.

In the two-dimensional case the asymptotic form of $\mathbf{E}(\mathbf{r}){ }_{t}$ for large $r$ is

$$
\mathbf{E}(\mathbf{r})_{t, \text { asymp }}=\left(\frac{2 \pi}{i k}\right)^{\frac{1}{2}} k \cos \theta^{\prime} r^{-\frac{1}{2}} e^{i k r} \mathbf{B}\left(k \sin \theta^{\prime}\right),
$$

where $\theta^{\prime}=\tan ^{-1}(x / z)$. From this follows

$$
\mathbf{S}_{10}\left(k_{x}\right)=\left(\frac{i k}{2 \pi}\right)^{\frac{1}{2}} a_{0}^{-1} \gamma^{-1} r^{\frac{1}{2}} e^{-i k r} \mathbf{E}(\mathbf{k} r / k)_{t, \text { asymp }}
$$

for $k_{x}^{2}<k^{2}$, where $\mathbf{k}=k_{x} \mathbf{e}_{x}+\gamma \mathbf{e}_{2}$.

The power radiation pattern becomes

$$
p=\pi(\epsilon / \mu)^{\frac{1}{2}} k \cos ^{2} \theta^{\prime} \mathbf{b} \cdot \overline{\mathbf{b}}
$$

where $\mathbf{b}$, the complete vectorial spectrum in the twodimensional case, is $\mathbf{b}=b_{x} \mathbf{e}_{x}+b_{z} \mathbf{e}_{z}$ and $b_{z}=-k_{x} b_{x} \gamma^{-1}$.

The two-dimensional forms of equations such as (18), (32), and (46) may be written without difficulty. [The equation corresponding to (32) is written as (53) in the text.] 


\section{References and Notes}

[1] For a general description of this work, see W. Culshaw, J. M. Richardson, and D. M. Kerns, Precision millimeter-wave interferometry at the U.S. National Bureau of Standards, Proc. of the Symposium on Interferometry at the National Physical Laboratory, Teddington, Middlesex, England (June 1959).

[2] A preliminary solution to the problem posed here using scalar waves and an asymptotic expansion of the reflection integral was obtained by Dayhoff (unpublished National Bureau of Standards Office of Basic Instrumentation report. This office supported the earlier phases of this work.)

[3] Microwave versions of the partially reflecting plates of the optical Fabry-Perot are discussed in the paper cited in note 1 ; see also W. Culshaw, Trans IRE, MTT-y, 221 (1959).

[4] See, e.g., J. A. Stratton, Electromagnetic theory, p. 361ff (McGraw-Hill Book Co., New York, N.Y., 1941).

[5] See e.g., B. Friedman, Principles and techniques of applied mathematics (John Wiley \& Sons, New York, N.Y., 1956).
[6] D. M. Kerns, Basis of the application of network equations to waveguide problems, J. Research NBS, 42, 515 (1949).

[7] This can be derived formally from (14) by application of methods originally devised for single integrals. For these methods see e.g., H. and B. S. Jeffreys, Methods of mathematical physics (Cambridge Univ. Press, 1950). For discussion of multiple integrals see D. S. Jones and M. Kline, Asymptotic expansion of multiple integrals and the method of stationary phase, N.Y.U., Inst. Math. Sci., Research Report No. EM-100 (1956).

[8] Discussed briefly in the reference cited in note 1.

[9] A convenient reference is J. A. Stratton, op. cit., Chap. 8.

[10] This is readily derivable from, e.g., eq (26), p. 578, in Stratton, op. cit.

[11] H. and B. S. Jeffreys, op. cit., p. 501ff.

[12] R. Courant and D. Hilbert, Methods of mathematical physics, 1st English Ed., p. 468 (Interscience Publishers, Inc., New York, 1953).

Boulder, Colo.

(Paper 64B1-15) 